\title{
ANÁLISIS DE LOS CONTENIDOS DE EXPRESIÓN CORPORAL IMPARTIDOS EN LA FORMACIÓN INICIAL DE LOS DOCENTES DE PRIMARIA
}

\author{
(ANALYSIS OF THE CONTENTS OF PHYSICAL EXPRESSION \\ IN THE PRE-SERVICE TRAINING OF PRIMARY TEACHERS)
}

Galo Sánchez

Marga López

Universidad de Salamanca

DOI: 10.5944/educXX1.20058

\begin{abstract}
Cómo referenciar este artículo/How to reference this article:
Sánchez, G. y López, M. (2019). Análisis de los contenidos de expresión corporal impartidos en la formación inicial de los docentes de primaria. Educación XX1, 22(1), 425-447, doi: 10.5944/ educXX1.20058

Sánchez, G. \& López, M. (2019). Análisis de los contenidos de expresión corporal impartidos en la formación inicial de los docentes de primaria. [Analysis of the contents of physical expression in the pre-service training of primary teachers]. Educación XX1, 22(1), 425-447, doi: 10.5944/ educXX1.20058
\end{abstract}

\section{RESUMEN}

El presente artículo aborda el análisis de la formación inicial de los docentes de Primaria en la disciplina de Expresión Corporal con el objetivo de contrastar los contenidos planificados en las guías docentes para su enseñanza con los elementos que los autores y autoras del ámbito consideran claves en la técnica del movimiento expresivo. Desde la complementariedad metodológica se realizó un estudio descriptivo y documental de las asignaturas impartidas en los estudios de Grado de Maestro/a de Primaria de las universidades públicas españolas. Dada la diversidad y heterogeneidad existente, se utilizó el criterio de seleccionar únicamente aquellas asignaturas en cuyas denominaciones apareciesen, al menos, dos de los siguientes términos: "expresión, corporal, movimiento, expresivo». Se analizaron los aspectos generales de las asignaturas de la muestra, como número de créditos o porcentaje presencial y teórico, así como el apartado «Contenidos» de las guías docentes. Los resultados obtenidos 
reflejan una tendencia actual a considerar la Expresión Corporal como una enseñanza prescindible en la formación al disponer únicamente de 6 créditos ECTS de carácter optativo en el cómputo global del Grado. Las asignaturas, desde un enfoque más práctico que teórico, presentan unos contenidos de naturaleza variada sin apenas referencias a la técnica propia de la disciplina, orientándose a la enseñanza de técnicas diversas de danza, dramatización, mimo o actividades de tipo lúdico como juegos, canciones y acrosport. También son escasas las referencias a la creatividad o a los procesos creativos en el balance total de las guías. Las conclusiones ofrecen unas directrices para guiar la didáctica de la Expresión Corporal en los estudios de Grado con el objetivo de transmitir al alumnado los conocimientos necesarios para implementar la disciplina como aprendizaje de valor en las escuelas.

\section{PALABRAS CLAVE}

Educación del movimiento; Escuelas de Magisterio; Investigación sobre programas de estudio; Relación entre teoría y práctica.

\section{ABSTRACT}

This paper deals with the analysis of pre-service training of primary teachers in the area of physical expression. Its aim is to compare the contents of the teaching guides with the key concepts which are considered essential by the scholars in the field of the expressive movement technique. From a twofold methodological perspective, a descriptive and documental analysis of the subjects present in the primary school teaching degrees taught in Spanish state universities was carried out. Due to the existing diversity and heterogeneity of these programs, only the subjects where the concepts of «corporal, expression, movement, expressive» appeared were selected. General aspects of the subjects such as number of credits, theoretical and classroom teaching percentage, and the "Contents» section of the teaching guides were included. The results obtained show a current trend to consider physical expression as a dispensable area in the training of prospective primary teachers as it only has 6 ECTS credits in an elective course, out of all the credits of the degree. The subjects, from a more practical than theoretical approach, present contents of a different nature with hardly any references to the own technique of the discipline, pointing to the teaching of various techniques of dancing, dramatization, mime or playful activities such as games, songs and acrosport. References to creativity or to the creative processes are also scarce in the total balance of the guides. Our conclusions offer some guidelines to lead the didactics of body language in the degrees with the aim of transmitting students the knowledge needed to implement the discipline as a learning of unquestionable value in schools.

\section{KEY WORDS}

Movement Education; Schools of Education; Curriculum Research; Theory Practice Relationship. 


\section{INTRODUCCIÓN}

La Expresión Corporal (EC en adelante) es una disciplina que cuenta con un corpus teórico propio y una fundamentación epistemológica consolidada gracias a las numerosas investigaciones y publicaciones especializadas que en los últimos años han abordado su estudio, tanto desde su faceta social y educativa (Sánchez, Coterón, Padilla, Llopis, y Montávez, 2008), como desde su uso para la formación y preparación actoral (Ferrari, 2014, 2017; Pérez de Amézaga, 2015). Sin embargo, el hecho de que se encuentre inmersa en diversos ámbitos como el pedagógico, psicológico, terapéutico, social y artístico (Pérez de Amézaga, 2015) tiene como consecuencia que su estudio se afronte desde orientaciones bastante dispares entre sí, generando la sensación de que no existe un acuerdo común entre los diferentes profesionales sobre cuáles deben ser sus principios y contenidos, e incluso sobre qué es y no es EC (Gil, 2016). En este sentido se expresa Quintana en Gil (2016, p. 58) cuando afirma que «lo que para un colectivo es Expresión Corporal para otro no lo es tanto». En opinión de Learreta (2004) esta falta de consenso es un problema que debe ser resuelto para permitir la construcción de un soporte teórico claro.

Una de las razones que, a nuestro parecer, alimenta esta confusión a la hora de delimitar un campo de estudio propio de la EC, es su presencia como asignatura en diversas enseñanzas superiores, al encontrarla integrada tanto en planes de estudio de Escuelas y Conservatorios de Arte Dramático como en determinadas titulaciones universitarias de Grado, vinculada normalmente a la Educación Física (EF en adelante). Este hecho, puede llevar a cuestionar cuál es en realidad la propia naturaleza y esencia de la EC, y si desarrollar destrezas expresivas a través del movimiento con fines creativos es patrimonio único de las enseñanzas artísticas. En este sentido, nuestra postura, y coincidiendo con Coterón y Sánchez (2010) es clara: la EC tiene un importante valor pedagógico como disciplina que favorece el desarrollo de recursos en el alumnado de tipo motriz, expresivo y creativo, por lo que debe quedar integrada y contemplada en la acciones educativas a realizar en la escuela; en esta misma línea de pensamiento sobre el binomio EC y educación, Schinca (2011a) expresa su potencial para estimular en niños y adolescentes ámbitos de la personalidad, como el cognitivo, sensoperceptivo, motriz, comunicativo y creativo-imaginativo.

En este sentido, si nos aproximamos a la realidad de la disciplina en la educación obligatoria, la presencia de la EC en estas etapas viene marcada en España por la Ley General de Educación de 1970, que supuso el inicio de su andadura como materia curricular al introducir por primera vez en el sistema educativo sus contenidos dentro del área de la Expresión Dinámica; posteriormente la LOGSE (1990) impulsaría definitivamente 
su integración como contenido propio de la Educación Física (Coterón y Sánchez, 2010; Montávez, 2012). Por tanto, en el contexto de la formación inicial del profesorado de Primaria y Secundaria, la EC queda integrada en los planes de estudio de las titulaciones de Grado en Educación Primaria y de Ciencias de la Actividad Física y del Deporte (Gil, 2016; Montávez, 2014), con una mayor presencia y peso en esta última, ya que la mayoría de titulaciones ofertadas por las universidades españolas presentan en sus planes de estudio, al menos, una asignatura vinculada a la EC con 6 créditos ECTS de carácter obligatorio (Conesa, 2017).

Por último, centrando la atención en la titulación de Grado en Educación Primaria, la modificación y reajuste de los títulos como consecuencia de la convergencia del Espacio Europeo de Educación Superior -EEES- de 2010, provocaron en opinión de Romero y Chivite (2013), un retroceso para el área de EF con la desaparición de la Especialidad e implantación de la actual Mención. Esta transición supuso la pérdida de la figura del Maestro/a especialista en EF y una reducción considerable de créditos específicos, pasando de los 206 créditos propios que disponía la especialidad, de los cuales 120 estaban destinados a una formación específica, a contar únicamente entre 30 y 60 créditos propios para la mención (Montávez, 2012); irremediablemente, la asignatura de EC se verá también afectada, pasando de ser planteada por lo general con carácter obligatorio (Cuéllar y Pestano, 2013) a quedar integrada en el conjunto de asignaturas optativas en la mención de EF, como será revisado a lo largo del presente artículo.

Una primera revisión a los planteamientos generales de la disciplina en la formación inicial del docente de Primaria nos lleva a compartir la reflexión de Gil (2016) cuando afirma que la asignatura de EC impartida en el Título de Grado en Ciencias del Deporte ha servido durante años como cajón de sastre de contenidos muy diversos. Creemos que la situación actual de la materia en la formación de los docentes de Primaria es equiparable a la descrita por el autor. Bajo nuestro criterio, a la escasa presencia curricular de la EC en el Grado hay que añadir la gran heterogeneidad y disparidad de contenidos contemplados en la enseñanza de la misma que generan confusión sobre su verdadero sentido y utilidad.

Existen autores como Pérez de Amézaga (2015) que desde las Artes Escénicas, revindican y defienden la identidad propia de la EC y su independencia de otras disciplinas artísticas como la Danza, el Mimo o el Clown. Sin embargo, dentro de los diversos ámbitos ya comentados donde se inserta la EC, la naturaleza tanto de los objetivos a alcanzar como del contexto educativo, abre un abismo en lo referente a los procesos de enseñanza planteados en cada uno de ellos. Conseguir adaptar a cada uno de estos contextos una técnica compartida para la enseñanza de la EC 
procurando evitar que quede desvirtuada en exceso por el uso de otras disciplinas, es un reto al que habría que hacer frente.

Si analizamos el primer nivel de concreción curricular, en el Área de EF de Primaria las disciplinas relacionadas con el movimiento y la expresión van de la mano. En la actual Ley Orgánica para la Mejora de la Calidad Educativa (LOMCE) de 2013 y, concretamente en el Real Decreto 126/2014 por el que se establece el currículo de Primaria, la orientación es diseñar la programación de la asignatura de EF organizando los contenidos en relación a las siguientes situaciones motrices: a) acciones individuales en entornos estables, que incluye entre otras cuestiones el desarrollo del esquema corporal; b) de oposición como juegos de uno contra uno, o la lucha; c) de cooperación, con o sin oposición a través de juegos de grupo, deportes colectivos, etc; d) de adaptación al entorno físico mediante marchas, excursiones, juegos en la naturaleza, etc; e) de indole artística o de expresión, que integra a las disciplinas escénicas como la expresión corporal, las danzas, el juego dramático y el mimo.

Ciertamente, la EC en el ámbito escolar y por extrapolación, en la formación del docente de Primaria, ha encontrado un camino propio que la aleja inexorablemente de la EC impartida en las Escuelas Superiores de Arte Dramático cuyo objetivo es la formación actoral. Desde esta perspectiva, nuestro criterio es que los contenidos de la disciplina deben diseñarse con la intención de aportar un valor al área de la EF escolar y al aprendizaje competencial del alumnado de Primaria, pero sin perder el marco teórico y epistemológico que la sustenta.

Centrándonos en la formación inicial del maestro/a, para Motos y G. Aranda «la expresión surge de la dialéctica equilibrada entre la creatividad y la técnica, entre la espontaneidad y la regla establecida» (2005, p. 14). Compartimos la reflexión de los autores y consideramos imprescindible para la didáctica de la Expresión Corporal en los Grados aplicar una técnica coherente que desarrolle en el alumnado destrezas y habilidades personales en la búsqueda de la expresividad a través del movimiento y que no reduzca la práctica a una experiencia lúdica sin orden ni objetivo pedagógico claro.

En concreto, la técnica de la Expresión Corporal debe orientarse a facilitar la exploración del mundo interno para manifestar una subjetividad mediante la utilización de un lenguaje corporal propio creado por la persona, con fines creativos y comunicativos (Schinca, 2010, 2011b; Coterón y Sánchez, 2010). La experiencia expresiva así planteada queda organizada a través de una técnica de movimiento propia sistematizada por los profesionales del ámbito que el futuro docente debe conocer, experimentar 
e interiorizar; sin el conocimiento de dicha técnica, la puesta en práctica de la EC en la escuela está abocada al fracaso (Lago y Espejo, 2007).

En este sentido, Coterón y Sánchez (2010) articulan la dimensión expresiva del movimiento en función de los siguientes ejes: Expresividad, mediante la toma de conciencia y manifestación del mundo interno; Comunicación con los demás a través del lenguaje corporal; Creación, que implica al pensamiento asociativo y divergente; Estética, movimiento expresivo con una finalidad escénica o de creación de un producto estético.

Montávez (2012), en un modelo adaptado del anterior, engloba dentro de la Expresividad el estudio de las Bases físicas para la toma de conciencia y de las Bases expresivas para la vivencia de la interrelación del cuerpo en movimiento en el espacio y en el tiempo con el objetivo de desarrollar las Habilidades expresivas para la comunicación del mundo interior.

La técnica elaborada a lo largo de los años por Schinca que anteriormente estaba estructurada en función de la toma de conciencia del cuerpo, del espacio, del tiempo y de la relación cuerpo-espacio-tiempo (Schinca, 2010), ha sido recientemente revisada por Ruiz (2011) y Ferrari $(2014,2017)$ quedando los contenidos a desarrollar divididos en dos bloques: por un lado las Bases físicas, que implica la toma de conciencia corporal y la sensibilización de la persona a los estímulos; y por otro lado la Expresión, a través del trabajo con los factores expresivos, su integración en los procesos expresivos y su desarrollo en los temas universales de expresión. En el método diseñado por Schinca, la comunicación y la creatividad quedan implícitas en la práctica expresiva, y puede estar orientado a una finalidad escénica con la creación de una producto final en forma de espectáculo de Teatro de Movimiento (Ruiz, 2011).

Por último, es interesante destacar la propuesta realizada por Gil (2016), que tras una revisión exhaustiva en la búsqueda de unos contenidos para la EC que organicen y aporten coherencia a la asignatura impartida en los Grados en Ciencias del Deporte, estructura finalmente el trabajo del lenguaje corporal en elementos corporales, que incluyen a su vez las Bases físicas y las Habilidades expresivas, y en elementos espaciales y temporales.

En definitiva, tras revisar los principios que deben vertebrar la enseñanza de la técnica del movimiento expresivo señalados por los autores y autoras, encontramos una serie de «lugares comunes» que quedan sintetizados en la Tabla 1: 
Tabla 1

Elementos que articulan la técnica de EC según autor/a

\begin{tabular}{c|l}
\hline Autor/es & \multicolumn{1}{c}{ Articulación de la Expresión Corporal } \\
\hline $\begin{array}{c}\text { Coterón y Sánchez } \\
\text { (2010, p. 121) }\end{array}$ & $\begin{array}{l}\text { Expresividad: Toma de conciencia y manifestación del mundo interno. } \\
\text { Exteriorización de ideas, estados de ánimo, sentimientos y emociones } \\
\text { Comunicación: } \text { Proceso intencional de interrelación con los demás a tra- } \\
\text { Creación: Proceso de construcción basado en el pensamiento asociativo y } \\
\text { divergente y enfocado al logro de resultados originales y elaborados. } \\
\text { Estética: Materialización del proceso creativo colectivo a través de un } \\
\text { producto con rasgos reconocibles de equilibrio, composición y armonía. }\end{array}$ \\
\hline $\begin{array}{c}\text { Montávez } \\
\text { (2012, p. 41-47) }\end{array}$ & $\begin{array}{l}\text { Bases físicas: tomar conciencia de la realidad corporal } \\
\text { Bases expresivas: desarrollo de las calidades de movi- } \\
\text { miento mediante el trabajo de cuerpo-espacio-tiempo. } \\
\text { Habilidades expresivas: Expresión del mundo interior de } \\
\text { la persona a través del movimiento significativo y simbó- } \\
\text { lico. }\end{array}$ \\
\cline { 2 - 3 }
\end{tabular}

Comunicación: Lenguaje gestual y corporal que genera un lenguaje propio para la comunicación a través del cuerpo, el espacio y el tiempo

Creatividad: Desarrollo de procesos creativos

Estética: Desarrollo de la sensibilidad estética

\begin{tabular}{|c|c|c|}
\hline \multirow[b]{2}{*}{$\begin{array}{l}\text { Schinca } \\
\text { (Ferrari, 2014, p. 85; } \\
\text { Ferrari, 2017, p. 61) }\end{array}$} & Bases Físicas & Toma de conciencia del cuerpo \\
\hline & Expresión & $\begin{array}{l}\text { Factores expresivos: Espacio, tiempo, fuerza } \\
\text { Procesos expresivos: Calidades de movimiento, modos de } \\
\text { movimiento, acciones básicas de esfuerzo } \\
\text { Temas universales de expresión: Caminar, animalidad, } \\
\text { movimiento y sonido, cuerpo y objeto. }\end{array}$ \\
\hline \multirow{3}{*}{$\begin{array}{c}\text { Gil } \\
(2016, \text { p. 332) }\end{array}$} & $\begin{array}{l}\text { Elementos } \\
\text { corporales }\end{array}$ & $\begin{array}{l}\text { Bases físicas: Control estático, dinámico, tonicidad y res- } \\
\text { piración } \\
\text { Habilidades expresivas: Cuerpo emocional, simbólico y } \\
\text { adjetivado }\end{array}$ \\
\hline & $\begin{array}{l}\text { Elementos } \\
\text { espaciales }\end{array}$ & $\begin{array}{l}\text { Enfoque estructural: límites y barreras del espacio; ilumi- } \\
\text { nación; disposición de los objetos. } \\
\text { Enfoque escénico: significación emocional de los niveles, } \\
\text { trayectorias y formaciones para un espectador externo. } \\
\text { Enfoque social: para la interacción con demás, orienta- } \\
\text { ción, distancia y distribución del espacio }\end{array}$ \\
\hline & $\begin{array}{l}\text { Elementos } \\
\text { temporales }\end{array}$ & $\begin{array}{l}\text { Ritmo corporal: duración, velocidad, intensidad, pausa } \\
\text { Pulso, acento, compás. } \\
\text { Composición expresiva: organizar secuencias de movi- } \\
\text { mientos en función de un ritmo }\end{array}$ \\
\hline
\end{tabular}


En consecuencia con lo expuesto hasta el momento, la investigación que aquí presentamos se planteó con el propósito de analizar en qué medida los elementos señalados por los teóricos como elementales en la enseñanza de la técnica de la EC y sintetizados en la Tabla 1 se encontraban presentes en las Guías Didácticas de las asignaturas vinculadas directamente con la disciplina en la formación inicial de los futuros docentes de Primaria. Junto con el análisis de dichos elementos, se revisaron también los aspectos más generales de dichas asignaturas para obtener una comprensión más amplia de su realidad dentro de los Planes de Estudios en los títulos de Grado de Educación Primaria.

\section{METODOLOGÍA}

Con el fin de abordar el problema de investigación planteado, se llevó a cabo un estudio documental de las guías docentes de las asignaturas de Expresión Corporal que se imparten en los Grados de Maestro/a de Primaria. Desde la complementariedad metodológica, se utilizó el análisis de contenido cuantitativo y cualitativo para inferir cómo quedan integrados en las guías los elementos técnicos de la disciplina extraídos como referentes del marco teórico establecido; también fueron analizados los aspectos más generales de las asignaturas, como la variedad en las denominaciones, porcentaje de horas presenciales o número de créditos. Para la realización del análisis de contenido de las guías se utilizó el programa informático Atlas.ti.

\section{Selección de la muestra}

La población para la extracción de la muestra estuvo compuesta por el total de asignaturas impartidas en los Grados de Maestro/a de Primaria de las universidades públicas españolas que integran la Expresión Corporal entre sus contenidos.

Cuéllar y Pestano (2013) clasifican estas asignaturas en 8 grupos: Expresión Corporal; Deportes; Juegos y Recreación; Fundamentos teóricos de la Educación Física; Didáctica de la Educación Física; Ciencias médicas y de la Salud; Psicomotricidad; Otros.

Ante la gran diversidad y heterogeneidad de nomenclaturas existentes, para la selección de la muestra se utilizó el siguiente criterio:

- Asignaturas en cuya denominación aparecieran, al menos, dos de los siguientes términos: movimiento, expresivo, expresión, corporal. 
Por tanto asignaturas como: Formación rítmica y danza o Actividades físico-expresivas, quedaron excluidas del estudio.

La selección de la muestra tuvo lugar durante el mes de julio de 2017 a través del buscador de títulos universitarios del Ministerio de Educación, Cultura y Deporte (MECD) con los siguientes parámetros de búsqueda: Educación > Formación de docentes de enseñanza primaria > Pública > Todas las CCAA, obteniendo como resultado 79 registros donde se imparte el Grado, de los cuales 35 registros cumplían el criterio - 44,3\% del totalquedando la muestra compuesta finalmente por 39 asignaturas y 24 guías docentes (varias de las asignaturas objeto de estudio comparten guía docente al ofertarse en centros que pertenecen a la misma universidad).

\section{Procedimiento}

Para garantizar la validez, siguiendo las consideraciones al respecto realizadas por Ballesteros y Mata (2014), se tuvo en cuenta tanto la rigurosidad y transparencia en la planificación de la investigación, como la triangulación de metodologías para el análisis de contenido de las guías, empleando técnicas diversas como la exploración cuantitativa y cualitativa de términos o la construcción de un sistema de categorías para el análisis deductivo.

En consecuencia, se planificaron las siguientes fases para el análisis:

1. Un primer análisis descriptivo de las asignaturas objeto de estudio para obtener una visión general de su presencia en el Grado. Se revisaron las 39 asignaturas de la muestra y se analizaron bajo un enfoque cuantitativo los siguientes aspectos: denominaciones, número de créditos, carácter, carga presencial y carga teórica.

2. Tras esta primera revisión de los datos, se analizó el contenido de las 24 guías docentes de la muestra desde dos enfoques metodológicos:

- Cuantitativo: con el fin de obtener una primera valoración, se estudió la frecuencia de aparición de términos de interés mediante un acercamiento prospectivo a la guía en su totalidad.

- Cualitativo: en primer lugar para profundizar en el contexto de aparición de los términos; posteriormente el interés del análisis se centró en el apartado específico de los «Contenidos». 
El sistema de categorías en función del cual se articuló el análisis queda recogido en la Tabla 2:

Tabla 2

Sistema de categorías para el análisis de contenido

\begin{tabular}{c|l}
\hline $\begin{array}{c}\text { Categoría 1 } \\
\begin{array}{c}\text { Toma de conciencia } \\
\text { de uno mismo }\end{array}\end{array}$ & $\begin{array}{l}\text { Desarrollo de la conciención corporal. Construcción del es- } \\
\text { quema corporal. Trabajo propioceptivo, exteroceptivo, explo- } \\
\text { ración de sensaciones corporales provocadas por el cuerpo en } \\
\text { movimiento. Sensopercepción, afinación de los sentidos. }\end{array}$ \\
\hline $\begin{array}{c}\text { Categoría 2 } \\
\text { Comunicación }\end{array}$ & $\begin{array}{l}\text { Trabajo con los factores expresivos: espacio, tiempo, ener- } \\
\text { gía, fuerza. Calidades de movimiento. Acciones básicas de es- } \\
\text { fuerzo. Interrelación con los demás a través del lenguaje cor- } \\
\text { poral expresivo simbólico y significativo. }\end{array}$ \\
\hline $\begin{array}{c}\text { Categoría 3 } \\
\text { Creatividad }\end{array}$ & $\begin{array}{l}\text { Creación de un lenguaje corporal propio, simbólico, abstracto, } \\
\text { significativo. Desarrollo de la creatividad, improvisación de } \\
\text { movimiento. Realización de un proyecto de creación, trabajo } \\
\text { expositivo, pieza escénica. }\end{array}$ \\
\hline $\begin{array}{c}\text { Categoría 4 } \\
\text { Disciplinas artísticas }\end{array}$ & $\begin{array}{l}\text { Danza, dramatización, teatro, mimo, clown. Trabajo coreográ- } \\
\text { fico. }\end{array}$ \\
\hline $\begin{array}{c}\text { Categoría 5 } \\
\text { Otros contenidos }\end{array}$ & $\begin{array}{l}\text { Contenidos que no son propios ni relevantes para la práctica } \\
\text { de la EC en la escuela. }\end{array}$ \\
\hline
\end{tabular}

\section{Resultados del análisis}

\section{Estudio descriptivo aspectos generales}

Las Tablas 3, 4, 5 y 6 reflejan los aspectos generales de la asignatura en cuanto a denominaciones, número de créditos, carácter, carga presencial y teórica. 
Tabla 3

Denominaciones de las asignaturas: Frecuencias absolutas (Fi) y relativas (fi)

\begin{tabular}{lrr}
\hline \multicolumn{1}{c}{ Nombre asignatura } & Fi & fi \\
\hline Expresión corporal & 12 & $30,80 \%$ \\
Expresión y comunicación corporal & 8 & $20,50 \%$ \\
Imagen, percepción, expresión y comunicación corporal & 5 & $12,80 \%$ \\
Expresión vocal y corporal & 3 & $7,70 \%$ \\
Percepción, movimiento y expresión & 2 & $5,10 \%$ \\
L'expressió corporal i la dansa & 2 & $5,10 \%$ \\
Expresión corporal y juegos & 2 & $5,10 \%$ \\
Expresión corporal y comunicación & 1 & $2,50 \%$ \\
Expresión corporal e adaptacións curriculares & 1 & $2,50 \%$ \\
Movimiento expresivo y creativo & 1 & $2,50 \%$ \\
Movimiento expresivo, danza y ritmo & 1 & $2,50 \%$ \\
La expresión corporal como recurso expresivo y comunicativo & 1 & $2,50 \%$ \\
\hline
\end{tabular}

Tabla 4

Número de créditos y tipo de asignatura

\begin{tabular}{ccrlrr}
\hline Créditos & Fi & \multicolumn{1}{c}{ fi } & \multicolumn{1}{c}{ Carácter } & Fi & \multicolumn{1}{c}{$\mathbf{f i}$} \\
\hline 6 & 33 & $84,6 \%$ & Optativa & 37 & $95,0 \%$ \\
4,5 & 4 & $10,3 \%$ & Básica de mención & 1 & $2,5 \%$ \\
3 & 2 & $5,1 \%$ & Obligatoria de mención & 1 & $2,5 \%$ \\
\hline & 39 & $100,0 \%$ & & 39 & $100,0 \%$ \\
\hline
\end{tabular}

Tabla 5

Carga presencial

\begin{tabular}{crr}
\hline Carga presencial & Fi & \multicolumn{1}{c}{ fi } \\
\hline$<\operatorname{del} 40 \%$ & 12 & $32,4 \%$ \\
$40,00 \%$ & 22 & $59,5 \%$ \\
$>\operatorname{del} 40 \%$ & 3 & $8,1 \%$ \\
\hline & $37^{1}$ & $100,0 \%$ \\
\hline
\end{tabular}


Tabla 6

Carga teórica

\begin{tabular}{crr}
\hline Carga teórica $^{2}$ & Fi & fi \\
\hline$<\operatorname{del} 10 \%$ & 15 & $46,9 \%$ \\
$10 \%-20 \%$ & 12 & $37,5 \%$ \\
$20 \%-30 \%$ & 5 & $15,6 \%$ \\
\hline & $32^{3}$ & $100,0 \%$ \\
\hline
\end{tabular}

En líneas generales se observa que las asignaturas adoptan la denominación más común de Expresión Corporal (30'80\%), seguida de Expresión y Comunicación Corporal (20,5\%); se incluyen en los Planes de Estudios de los Grados en la Mención de Educación Física como asignaturas de carácter optativo (95\%), con carga lectiva de 6 créditos ECTS $(84,6 \%)$, con un porcentaje presencial del $40 \%$ o inferior en la mayoría de casos $(92 \%)$, y en el planteamiento prima la práctica frente a la teoría, siendo la carga teórica inferior al $10 \%$ en el $47 \%$ de las guías.

\section{Análisis de contenido}

Frecuencia y contexto de aparición de términos

La Tabla 7 recoge un extracto del análisis prospectivo mediante nube de palabras; este análisis ofrece una visión general de la frecuencia de aparición —en frecuencias absolutas- de determinados términos considerados de interés para el estudio ${ }^{4}$.

Tras este primer análisis como punto de partida, el interés posterior se centró en el contexto concreto en que se encontraban inmersos algunos de los términos, obteniendo que:

— «Técnica» (Fi: 9) y «Técnicas» (Fi: 42) se encuentran principalmente en las referencias bibliográficas utilizadas en las guías, o vinculadas a la metodología docente de la asignatura - especialmente en el apartado de la evaluación- y a las competencias y resultados del aprendizaje del alumnado de Grado. Únicamente dos guías hacen una mención explícita a la técnica/s de expresión corporal y técnicas del lenguaje expresivo. 
Tabla 7

Frecuencia de determinados términos presentes en las guías

Toma de conciencia

Sensopercepción:1 Sensibilidad: 6 Sensomotriz: 3 Sensorial:1 Sensoriales: 5 Explorar: 3 Observar: 2 Observación: 7 Percepción: 14 Perceptivo: 9 Emociones/ Emocions: 11

\section{Comunicación}

Comunicación: 129 Espacio/ espais: 30 Tiempo/ temps: 28 Ritmo/s: 30 Rítmicas: 8

Energía: 4 Peso: 7 Intensidad: 2 Calidad: 20 Calidades: 3

Proceso creativo

Creatividad y derivadas: 77 Creación/ Creació: 29 Composición:1 Montaje: 14 Espectáculos: 2 Improvisación: 15 Estético: 3 Estéticas: 1 Coreografía: 10 Coreografías: 6

\section{Disciplinas expresivas}

Danza/ Dansa/ Dança: 82 Danzas: 17 Bailes: 10 Baile: 3 Mimo: 5 Teatro:15 Clown: 6 Circenses: 1 Dramatización: 29 Musical: 43 Musicales: 8 Canciones: 16 Folclore/ Folklore: 4

\section{Otros contenidos}

Técnica: 9 Técnicas: 42 Acrosport: 6 Acrocoreográfico: 2 Deporte: 22 Deportes: 23 Juegos: 58 Juego: 26

— «Creatividad» y sus términos derivados (Fi: 77) los encontramos en las referencias bibliográficas de las guías y en los apartados de objetivos y competencias correspondientes a la asignatura. Seis guías no presentan referencias al término, aunque sí a alguna de sus derivaciones. Únicamente en dos guías «creativo» alude al «movimiento», relacionándose en la mayoría de casos con estilo, trabajo, proyecto, proceso o resultado.

- «Espacio» (Fi: 30) concierne en la mayoría de sus apariciones al cuerpo y al movimiento, por lo que es tratado como factor expresivo. En algunos documentos el término alude también a infraestructuras o espacios curriculares, pero en general, prima el sentido expresivo.

— «Tiempo» (Fi: 28) está vinculado por igual tanto con el movimiento como con el tiempo curricular de la asignatura.

- «Danza» (Fi: 82) se encuentra unida a disciplinas como el mimo o la dramatización y se presenta como una manifestación o actividad expresiva de carácter multifacético, que incluye bailes de salón, danza caribeña, popular, clásica, repertorio, danzas del mundo, folclore..etc.

— «Ritmo/s» (Fi: 30) está ligado a la danza, a bailes y coreografías, interiorización del ritmo para el movimiento, cuerpo y ritmo como medios de expresión corporal, ritmo musical como base del 
movimiento danzado. En gran medida el término se encuentra en el contexto de las referencias bibliográficas.

\section{Análisis cualitativo}

Se llevó a cabo el análisis específico del apartado de los Contenidos de las guías docentes, para clasificar la naturaleza de los mismos y valorar su aproximación a las directrices aportadas por los autores y reflejadas en el marco teórico establecido. Para este análisis se empleó un enfoque deductivo mediante la creación previa de un sistema de categorías extraídas de la revisión teórica; las familias de códigos resultantes del análisis fueron:

- Familia 1: Toma de conciencia, donde quedaron incluidas citas concernientes a las emociones, expresión de sentimientos, percepción del cuerpo y el movimiento, esquema corporal, habilidades y destrezas, coordinación, equilibrio, educación de los sentidos, educación sensomotriz, lateralidad, percepción espacio-temporal y a la relajación. Doce de los documentos analizados no presentaban contenidos relacionados con la toma de conciencia. Los códigos incluidos en esta familia fueron: Propiocepción-sensopercepción (12 citas) / Observación-reflexión (1cita) / Emociones (3 citas).

- Familia 2: Comunicación, compuesta por citas relacionadas en gran medida con el estudio del movimiento en el espacio y en el tiempo y que, en general, hacen referencia a las habilidades expresivas (cuerpo, espacio, tiempo, energía), a la comunicación a través del cuerpo, al ritmo como manifestación expresivo-educativa, a la intensidad de movimiento como factor expresivo, al gesto, la comunicación no verbal y a las posturas. Siete de los documentos analizados no presentan una mención explícita a elementos relacionados con esta familia. Los códigos que conformaron la familia fueron: Comunicación con los demás y el entorno (9 citas)/ Factores expresivos e integración (14 citas).

- Familia 3: Creatividad, cuyas citas hacen referencia al proceso creativo - de forma explícita solo en cuatro documentos-, a la producción escénica, creación de coreografías, la creatividad relacionada con la exploración, propuestas creativas en relación a la expresión vocal, propuestas creativas en un sentido general, a la actividad libre y creativa, técnicas de desarrollo creativo basadas en la improvisación. Un documento especifica el uso de la Sinéctica corporal como técnica para la creatividad. En trece de los documentos analizados no hay ninguna referencia explícita a la creatividad en el apartado de los contenidos. Los códigos que quedaron integrados en esta familia fueron: Creatividad (8 citas) / Proceso creativo (6 citas) / Vivencia/improvisación (3 citas). 
Con el fin de facilitar la clasificación posterior de los diferentes tipos de contenidos según su pertenencia a una u otra categoría, fueron considerados también los contenidos de carácter general y teórico, así como los que trataban aspectos relativos a otras disciplinas artísticas. Por último el código «No pertinentes» fue creado para incluir en él aquellos contenidos considerados no propios de la disciplina - como acrosport, gimnasia rítmica, bailes de salón, sevillanas, teatro de sombras, fitness-.

La Tabla 8 aporta una visión de la situación general de la presencia (P) o ausencia (A) de códigos en las diferentes familias por guía docente analizada:

Tabla 8

Presencia de códigos según guía

\begin{tabular}{cccccc}
\hline Guías & $\begin{array}{c}\text { Toma } \\
\text { conciencia }\end{array}$ & Comunicación & Creatividad & $\begin{array}{c}\text { No } \\
\text { pertinentes }\end{array}$ & D. artísticas \\
\hline 1 & $\mathrm{P}$ & $\mathrm{P}$ & $\mathrm{P}$ & $\mathrm{A}$ & $\mathrm{A}$ \\
2 & $\mathrm{P}$ & $\mathrm{P}$ & $\mathrm{P}$ & $\mathrm{P}$ & $\mathrm{P}$ \\
3 & $\mathrm{~A}$ & $\mathrm{P}$ & $\mathrm{A}$ & $\mathrm{A}$ & $\mathrm{P}$ \\
4 & $\mathrm{P}$ & $\mathrm{P}$ & $\mathrm{A}$ & $\mathrm{P}$ & $\mathrm{P}$ \\
5 & $\mathrm{P}$ & $\mathrm{P}$ & $\mathrm{P}$ & $\mathrm{P}$ & $\mathrm{P}$ \\
6 & $\mathrm{~A}$ & $\mathrm{~A}$ & $\mathrm{~A}$ & $\mathrm{P}$ & $\mathrm{A}$ \\
7 & $\mathrm{P}$ & $\mathrm{A}$ & $\mathrm{P}$ & $\mathrm{P}$ & $\mathrm{P}$ \\
8 & $\mathrm{~A}$ & $\mathrm{P}$ & $\mathrm{P}$ & $\mathrm{A}$ & $\mathrm{P}$ \\
9 & $\mathrm{~A}$ & $\mathrm{~A}$ & $\mathrm{~A}$ & $\mathrm{~A}$ & $\mathrm{~A}$ \\
10 & $\mathrm{~A}$ & $\mathrm{P}$ & $\mathrm{P}$ & $\mathrm{P}$ & $\mathrm{P}$ \\
11 & $\mathrm{P}$ & $\mathrm{A}$ & $\mathrm{A}$ & $\mathrm{A}$ & $\mathrm{A}$ \\
12 & $\mathrm{P}$ & $\mathrm{P}$ & $\mathrm{A}$ & $\mathrm{P}$ & $\mathrm{P}$ \\
13 & $\mathrm{P}$ & $\mathrm{A}$ & $\mathrm{P}$ & $\mathrm{P}$ & $\mathrm{P}$ \\
14 & $\mathrm{P}$ & $\mathrm{P}$ & $\mathrm{A}$ & $\mathrm{P}$ & $\mathrm{P}$ \\
15 & $\mathrm{P}$ & $\mathrm{P}$ & $\mathrm{P}$ & $\mathrm{P}$ & $\mathrm{P}$ \\
16 & $\mathrm{~A}$ & $\mathrm{P}$ & $\mathrm{A}$ & $\mathrm{A}$ & $\mathrm{A}$ \\
17 & $\mathrm{~A}$ & $\mathrm{P}$ & $\mathrm{A}$ & $\mathrm{P}$ & $\mathrm{P}$ \\
18 & $\mathrm{P}$ & $\mathrm{P}$ & $\mathrm{A}$ & $\mathrm{A}$ & $\mathrm{A}$ \\
19 & $\mathrm{~A}$ & $\mathrm{P}$ & $\mathrm{A}$ & $\mathrm{A}$ & $\mathrm{A}$ \\
20 & $\mathrm{P}$ & $\mathrm{P}$ & $\mathrm{P}$ & $\mathrm{P}$ & $\mathrm{P}$ \\
21 & $\mathrm{~A}$ & $\mathrm{~A}$ & $\mathrm{~A}$ & $\mathrm{P}$ & $\mathrm{P}$ \\
22 & $\mathrm{~A}$ & $\mathrm{P}$ & $\mathrm{P}$ & $\mathrm{A}$ & $\mathrm{P}$ \\
23 & $\mathrm{~A}$ & $\mathrm{~A}$ & $\mathrm{P}$ & $\mathrm{A}$ & $\mathrm{P}$ \\
24 & $\mathrm{~A}$ & $\mathrm{~A}$ & $\mathrm{~A}$ & $\mathrm{~A}$ & $\mathrm{~A}$ \\
\hline
\end{tabular}


En síntesis se observa que:

- Gran parte de los documentos revisados contienen elementos que pertenecen a las categorías comunicación y disciplinas artísticas (presentes en el 67\% de las guías en ambos casos), seguidos de los contenidos no pertinentes (54\% de las guías) y los relacionados con la toma de conciencia (50\%). Por último, un 46\% de las guías contienen elementos en la categoría creatividad.

- Dos documentos no contienen citas en ninguno de los aspectos valorados en la tabla 8; esto es debido a que presentan contendidos muy generales o de tipo teórico-currículo, leyes educativas, historia de la EC, etc.

- Solo uno de los documentos abarca las tres cuestiones señaladas como prioritarias por la fundamentación teórica, sin incluir además contenidos considerados «no pertinentes».

\section{CONCLUSIONES}

El análisis descriptivo refleja una tendencia a considerar la asignatura de Expresión Corporal como una materia opcional y de relevancia inferior en la formación del docente de Primaria, siendo de carácter optativo en la mayoría de casos estudiados. Por otro lado, este hecho es coincidente con una estructura educativa en Áreas donde el desarrollo motriz se afronta en la escuela desde una única asignatura que integra a su vez entre sus contenidos a la educación corporal expresiva.

De naturaleza fundamentalmente práctica, la asignatura se suele ofertar en los Planes de Estudios de los Grados con 6 créditos ECTS, y cuenta con un porcentaje de carga presencial del $40 \%$ o inferior, siendo superior solo en tres de los casos analizados.

Una primera aproximación al contenido de las guías deja traslucir que, efectivamente, la práctica es prioritaria frente a la teoría, hecho por otro lado en consonancia con la singularidad de la asignatura.

Sin embargo, es especialmente llamativo el escaso número de referencias en las guías docentes a dos conceptos que consideramos prioritarios:

Por un lado a la técnica concreta de expresión corporal, encontrando habitualmente la técnica ligada a otras disciplinas como la danza, la dramatización o haciendo referencia a «técnicas expresivas» sin especificar. De forma más común, «técnica» y «técnicas» se encuentran en los apartados de las referencias bibliográficas y en la metodología docente de la asignatura. 
Igualmente, llama la atención la escasa presencia del término creatividad en el estudio general de las guías, con tan solo 77 referencias a ella o a términos derivados frente a otros como evaluación, con 205 referencias, o juego/s con 84; y si analizamos el contexto en que se halla inserto el término este hecho es aún más llamativo. En este sentido, creatividad suele encontrarse en las referencias bibliográficas, y fuera de este apartado, se vincula en gran parte con las competencias que el alumnado de Grado debe adquirir. En dos guías creatividad o alguna de sus palabras derivadas como creativo/a o creativamente- no están presentes, y únicamente en dos de los documentos analizados creatividad hace referencia al movimiento.

En la mayoría de guías, la danza — con 112 referencias al término-, el ritmo -30 referencias - o la dramatización -29 referencias- son entendidas como parte de la propia esencia de la disciplina. La danza se presenta como una actividad de naturaleza indefinida o haciendo referencia a una gran variedad de técnicas dispares entre sí como danzas populares, danzas del mundo o danza clásica.

A este respecto, nuestra reflexión es que las técnicas de danza son diversas, y en la mayoría de casos complejas, lo que impide abarcar su enseñanza en el escaso tiempo curricular del que dispone la asignatura, si los alumnos además no cuentan con una formación previa. Por añadidura, son técnicas cuyos lenguajes están muy codificados, motivo que a nuestro parecer, se aleja del objetivo de la libre expresión pretendido desde la Expresión Corporal, que se diluye en esta mezcla de técnicas dispares entre sí. Por tanto, al incluir el aprendizaje de determinadas técnicas de danza como parte del temario, se corre el riesgo de que el resultado sea anecdótico y de que su cometido se reduzca al aprendizaje de algún baile específico regional, de salón, etc.- - que el docente enseña a los alumnos para cumplir con las exigencias de la evaluación. Y que en ese punto finalice su recorrido como aprendizaje. Este tipo de actividad lúdica, muy útil para conseguir la desinhibición inicial, a nivel pedagógico tiene poco valor, mucho más si se contempla el fin último de la asignatura que debe estar enfocado en todo momento a la adquisición por parte de los futuros educadores de conocimientos y destrezas que puedan poner en práctica en la escuela con el alumnado de primaria, receptor final de dichos contenidos.

La otra opción es entender la danza dentro del concepto de Expresión corporal-danza definido por Patricia Stokoe o del de «Danza creativa» que se rige por los preceptos de la Expresión Corporal empleados en la técnica de movimiento Schinca. En ese sentido el término abarca a la disciplina y puede ser aplicado, con las directrices correctas, tanto con el alumnado de Grado como con el de Primaria. Y tiene una utilidad y un importante valor educativo, al facilitar que los alumnos conozcan y experimenten las 
posibilidades expresivas de su cuerpo en movimiento, así como el empleo expresivo del espacio y del tiempo, desde la propia creación y vivencia. Por tanto, nuestra postura sobre el tema es que la danza debe tener cabida en la disciplina, pero entendida desde este enfoque.

Existen infinidad de disciplinas que pueden complementar y enriquecer la formación del docente a nivel corporal y expresivo como el Clown, que emplea la improvisación y utiliza ejercicios de calentamiento muy interesantes dirigidos a la desinhibición, muy útiles en momentos determinados con los grupos de estudiantes, especialmente en las primeras sesiones; o la danza contemporánea , con una técnica muy rica en trabajo propioceptivo y de improvisación de movimiento. El aprendizaje de estas técnicas siempre sumará y enriquecerá el bagaje corporal y los recursos del docente. Sin embargo, estas disciplinas artísticas y corporales tienen otros objetivos, otra didáctica, y son en definitiva «otra cosa».

Como ha sido ya comentado, las técnicas de movimiento como las utilizadas en la danza clásica o los bailes de salón tienen unos códigos cerrados y rígidos, con movimientos y secuencias establecidas, motivo por el cual, nuestra postura es que no deberían formar parte de una asignatura cuyo objetivo es dotar al futuro docente de la capacidad flexible de trabajar con el cuerpo de manera creativa, integral y armónica.

En relación al Ritmo, el trabajo expresivo con el factor tiempo planteado desde la Expresión Corporal abarca su estudio, pero al igual que ocurre con la danza, si dicho estudio se afronta desde la enseñanza de canciones a las que combinar el movimiento de una coreografía aprendida, nos encontramos de nuevo en el mismo callejón sin salida: se ha vuelto a desperdiciar la oportunidad preciosa que la disciplina ofrece para la creación y la expresión libre.

En definitiva, defendemos la enseñanza de una Expresión Corporal en la formación inicial de los maestros y maestras de Primaria que disponga de la capacidad de ser una disciplina integradora de saberes, con contenidos variados e interconectados entre sí, no encasillados en compartimentos estancos, donde la danza, la dramatización o la composición coreográfica tengan cabida, pero siempre desde el filtro que aporta la técnica propia de la disciplina que los autores del ámbito, desde enfoques que comparten similitudes y elementos en común, han establecido.

Nuestro criterio es no desaprovechar la oportunidad que la Expresión Corporal ofrece para sensibilizar, para que la persona conozca y profundice en su mundo interior y pueda expresarlo a través del movimiento. También creemos importante resaltar la capacidad de la disciplina para ser utilizada 
como herramienta de creación, para estimular la creatividad en los estudiantes y facilitar la vivencia de los procesos creativos. Con el fin de alcanzar estos objetivos, debería primar el aprendizaje significativo que profundiza en la toma de conciencia del cuerpo y de uno mismo para llegar a la comunicación y a la expresión creativa a través del movimiento, frente al aprendizaje por imitación y copia de técnicas diversas.

Creemos que hay que ser ambiciosos a la hora de diseñar los contenidos que formarán a los futuros docentes para que la Expresión Corporal contribuya realmente al aprendizaje de competencias en el alumnado de Primaria y no termine siendo en la programación de Educación Física una unidad didáctica de tipo lúdico sin demasiado valor educativo. Intentar ser efectivos debería ser la prioridad, que los esfuerzos estén dirigidos a proporcionar una base sólida a los estudiantes en el escaso tiempo curricular del que dispone actualmente la asignatura en las enseñanzas de Grado.

Ofrecer unos buenos cimientos en formación básica facilitará al estudiante del título aplicar los conocimientos aprendidos en su tarea profesional e inculcará el interés por el movimiento expresivo en los docentes para que puedan crecer progresivamente en la disciplina dentro del contexto de la formación continua.

Somos conscientes de una realidad: la difícil tarea que supone conseguir este equilibrio entre proporcionar una formación de contenidos sistematizados y generar el interés suficiente por la materia en los estudiantes en un espacio curricular de tan solo 6 créditos ECTS. Obviamente, la solución pasaría por aumentar la carga lectiva a las disciplinas artísticas y de movimiento expresivo en la formación inicial de los futuros educadores. Sin embargo, nuestra postura al respecto, y coincidiendo con Montávez (2012), es más ambiciosa: creemos que ha llegado el momento de reclamar una formación corporal expresiva de calidad dentro del ámbito formativo de posgrado, donde la enseñanza de la EC se pueda desarrollar en toda su plenitud para formar a docentes especializados en la disciplina; esto ayudaría a dotar de una mayor presencia curricular a la educación corporal creativa, casi inexistente en la actualidad, y a que la EC pudiese llegar a conformarse como una materia independiente de la EF. Quizá esta propuesta tome consistencia real en el contexto de los nuevos paradigmas educativos, si la demanda social de generar ciudadanos cada vez más innovadores, creativos y con capacidad de desarrollar su inteligencia intrapersonal llega a materializarse definitivamente en las aulas. Sin embargo, en el planteamiento actual, y sin necesidad de recurrir a situaciones utópicas, una de las posibilidades al alcance del profesorado de Expresión Corporal debería consistir en sistematizar el proceso de enseñanza-aprendizaje para componer una buena hoja de ruta que guíe en la didáctica de la materia y que impida perderse en contenidos vacuos. 


\section{NOTAS}

1 En 2 registros esta información no está disponible.

2 Se han tenido en cuenta: Sesiones magistrales, Clases expositivas, Clases teóricas, Lección magistral, Exposición verbal, Teoría.

3 Registros sin contabilizar: Clase teórico-prácticas. Clases participativas. En 3 registros la información no está disponible o es confusa.

4 El programa no reconoce el formato del documento 18 correspondiente a la guía de la asignatura «Imagen, percepción, expresión y comunicación corporal» por lo que no ha formado parte del conteo general. 


\section{REFERENCIAS BIBLIOGRÁFICAS}

Ballesteros, B. y Mata, P. (2014). Sentido y forma de la investigación cualitativa. En B. Ballesteros (Coord.), Taller de investigación cualitativa (pp.12-46). Madrid: UNED.

Conesa-Ros, E. y Angosto, S. (2017). Análisis del contenido de «expresión corporal» en los planes de estudio de grado en ciencias de la actividad física y el deporte en universidades españolas. Journal of Sport and Health Research. 9(2), 263-272.

Coterón, J. y Sánchez, G. (2010). Educación artística por el movimiento: la Expresión Corporal en Educación Física. AULA, Revista de Pedagogía de la Universidad de Salamanca. (16), 113-134.

Cuéllar, M.J. y Pestano, M.A. (2013). Formación del Profesorado en Expresión Corporal: planes de estudio y Educación Física. RETOS, Nuevas tendencias en Educación Física, deportes y recreación. 24, 123-128.

Ferrari, H. (2014). Schinca, teatro de movimiento. [Tesis Doctoral]. Madrid: Universidad Rey Juan Carlos).

Ferrari, H. (2017). Marta Schinca, precursora del teatro del movimiento. Vol I. Madrid: Editorial Fundamentos.

Gil, J. (2016). Los contenidos de la Expresión Corporal en el Título de Grado en Ciencias del Deporte. [Tesis Doctoral]. Madrid: Universidad Politécnica de Madrid.

Lago, P. y Espejo, A. (2007). El movimiento y la danza. Su importancia en el currículum de Primaria. Educación y futuro. (17), 149-163.

Learreta, B. (2004). Los contenidos de Expresión Corporal en el Área de Educación Física en Enseñanza
Primaria. [Tesis Doctoral]. Madrid: Universidad Complutense.

Ministerio de Educación, Cultura y Deporte (2017). Qué estudiar y dónde en la universidad (QUEDU). Recuperado de https://goo.gl/oVquET

Montávez, M. (2012). La expresión corporal en la realidad educativa. Descripción y análisis de su enseñanza como punto de referencia para la mejora de la calidad docente en los centros públicos de Educación Primaria de la ciudad de Córdoba. [Tesis Doctoral]. Córdoba: Universidad de Córdoba.

Motos, T. y G. Aranda, L. (2005). Práctica de la expresión corporal (3. ${ }^{\mathrm{a}}$ ed). Guadalajara: Naque Editora.

Pérez De Amézaga, A.M. (2015). Estudio del método Schinca de Expresión Corporal en la Escuela Superior de Arte Dramático de Asturias. [Tesis Doctoral]. Oviedo: Universidad de Oviedo.

Real Decreto 126/2014, de 28 de febrero, por el que se establece el currículo básico de la Educación Primaria. (B.O.E. n. ${ }^{\circ} 52$ de 1 de marzo de 2014).

Romero, M.R. y Chivite, M. (2013). Análisis de la presencia social de la Expresión Corporal. EC y Universidad. RETOS, Nuevas tendencias en Educación Física, deportes y recreación. 24, 184-188.

Ruiz, R. (2011). Fundamentos de la expresión corporal. Cuadernos del Estudio Schinca. 1(0), 3-15.

Sánchez, G., Coterón, J., Padilla, C., Llopis, A., y Montávez, M. (2008). La expresión corporal en el marco del EEES. Un proyecto de consolidación. En G. Sánchez, J. Coterón, J. Gil, y A. Sánchez (Coords.), El movimiento 
expresivo. II Congreso Internacional de Expresión Corporal y Educación (pp. 17-27). Salamanca: Amarú Ediciones

Schinca, M. (2010). Expresión corporal. Técnica y expresión del movimiento (4. ${ }^{a}$ ed.). Madrid: Editorial Wolters Kluwer España.
Schinca, M. (2011a). Arte, expresión corporal y psicomotricidad. Cuadernos del Estudio Schinca. 1(0), 31-36.

Schinca, M. (2011b). Manual de psicomotricidad, ritmo y expresión corporal (2. ${ }^{\mathrm{a}} \mathrm{ed}$.). Madrid: Editorial Wolters Kluwer Educación. 


\section{PERFIL ACADÉMICO Y PROFESIONAL DE LOS AUTORES}

Galo Sánchez. Profesor Titular de la Universidad de Salamanca en la Escuela Universitaria de Magisterio de Zamora en el área de Didáctica de la Expresión Corporal. Doctor en Ciencias de la Educación por la Universidad de Salamanca, Licenciado en Educación Física (INEF Universidad Politécnica de Madrid).

Marga López. Doctoranda en el Programa de Educación en la Universidad de Salamanca; Máster en Innovación e Investigación en Educación (UNED); Experta en Didáctica de la Expresión Corporal (Universidad Rey Juan Carlos).

Dirección de los autores: Universidad de Salamanca

Escuela Universitaria de Magisterio de

Zamora

Cardenal Cisneros, 34

Campus Viriato

49022 Zamora

E-mail: galo@usal.es

margalp@outlook.com

Fecha Recepción del Artículo: 15. Octubre. 2017

Fecha Modificación del Artículo: 20. Enero. 2018

Fecha Aceptación del Artículo: 17. Marzo. 2018 